\title{
Nonreciprocal and non-Hermitian material response inspired by semiconductor transistors
}

\author{
Sylvain Lannebère ${ }^{1}$, David E. Fernandes ${ }^{1}$, Tiago A. Morgado ${ }^{1}$, and Mário G. Silveirinha2* \\ 1 Instituto de Telecomunicações and Department of Electrical Engineering, \\ University of Coimbra, 3030-290 Coimbra, Portugal and \\ ${ }^{2}$ University of Lisbon - Instituto Superior Técnico and Instituto de Telecomunicações, \\ Department of Electrical Engineering, 1049-001 Lisboa, Portugal
}

\begin{abstract}
Here, inspired by the operation of conventional semiconductor transistors, we introduce a novel class of bulk materials with nonreciprocal and non-Hermitian electromagnetic response. Our analysis shows that material nonlinearities combined with a static electric bias may lead to a linearized permittivity tensor that lacks the Hermitian and transpose symmetries. Remarkably, the material can either dissipate or generate energy, depending on the relative phase of the electric field components. We introduce a simple design for an electromagnetic isolator based on an idealized "MOSFET-metamaterial" and show that its performance can in principle surpass conventional Faraday isolators due to the material gain. Furthermore, it is suggested that analogous material responses may be engineered in natural media in nonequilibrium situations. Our solution determines an entirely novel paradigm to break the electromagnetic reciprocity in a bulk nonlinear material using a static electric bias.
\end{abstract}


The Lorentz reciprocity law constrains the propagation of electromagnetic waves in conventional photonic platforms [1, 2]. For Hermitian systems, the Lorentz reciprocity is rooted in the linearity of Maxwell's equations and on their invariance under a time-reversal (TR) operation [3]. Generically, a nonreciprocal electromagnetic response requires either (i) breaking the TR symmetry with a suitable bias, or (ii) using nonlinear materials, or (iii) exploiting non-Hermitian physics.

The standard solution to break reciprocity is by using a static magnetic field bias. The magnetic field is odd under the TR operation and thereby may give rise to a gyrotropic nonreciprocal permeability in ferrimagnetic materials [1, 4] or a gyroelectric nonreciprocal permittivity in magnetized plasmas [5, 6]. Such material platforms are extensively used to realize optical isolators and circulators [4, 7], and due to their topological properties [8] may enable "one-way" propagation free of back-scattering [9-20]. However, the necessity of an external magnetic field is a major drawback for the integration of such components on a chip. Alternative magnetless solutions have been investigated in recent years. Such solutions can be sub-divided into two classes, depending if they preserve or not the linearity of Maxwell's equations.

For the first class, the material response is linear under normal conditions of operation. Such solutions include time-variant systems [21 35], systems with moving parts [36 38], systems with drifting electrons [39-47], and non-Hermitian platforms, e.g. PT-symmetric systems [48 50], active electronic systems [51 53] or optically pumped systems [54]. It is relevant to point out that time-modulations, the drift current bias or the velocity bias, all imply an explicitly broken TR symmetry. In contrast, as highlighted recently in Ref. [54], a non-Hermitian response can be compatible with the TR symmetry, but yet break the reciprocity. All the solutions in this first class require some external bias of the system.

The second class is formed by systems that exploit dynamic nonlinear effects and that have been implemented in photonic crystals [55 65] or using Fano resonances [66 69], PTsymmetry [70 72] or other mechanisms [73 77]. These systems are typically self-biased by the incoming wave. Thus, they require input signals with very large power, and generally speaking they cannot provide a robust optical isolation [77-79].

Here, inspired by the physics of transitors, we unveil a different opportunity to generate a strongly nonreciprocal and non-Hermitian linearized material response, which relies on the combination of a static electric bias with nonlinearities. Crucially, even though the material 
nonlinearity is essential to break the reciprocity, in our system the effective material response to weak dynamical signals can be assumed linear. This property may be understood with an analogy with semiconductor transistors, which are nonlinear devices biased by static fields, and then behave as linear systems (e.g., amplifiers) for relatively weak dynamical signals.

It is curious to note that the nonreciprocal responses of other well-known systems with a broken TR symmetry also rely on nonlinear effects that are linearized around a biasing point for small amplitude signals. For example the gyrotropic permeability of ferrimagnetic materials and the gyroelectric permittivity of magnetized plasmas result from the linearization of the magnetic torque and of the Lorentz force, respectively [1]. Similarly, the nonreciprocal response of materials with a drift current results from the linearization of the Boltzmann equation [5, 6, 80 82]. Furthermore, the linearization of optomechanical interactions is at the origin of the nonreciprocity reported in Refs. [24, 83, 84].

Here, inspired by this feature and by the operation of standard transistors, we propose a new paradigm to obtain a nonreciprocal linearized response in a bulk nonlinear material, which combines the benefits of the linearized systems cited above with the simplicity of the electric field bias, providing a unique platform for the study of non-Hermitian and nonreciprocal wave phenomena.

As a starting point, consider a standard MOSFET transistor, as depicted in Fig. 1(a)-(i). In a MOSFET, the voltage applied on the gate controls the height of the channel that connects the drain and the source $[1,85]$. Thus, the electric field along the $z$-direction controls the "polarizability" of the system along the $x$-direction. Let us imagine a metamaterial

(a)

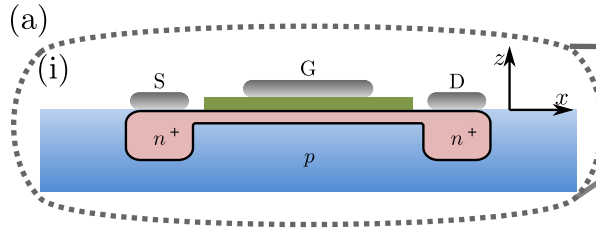

(ii)

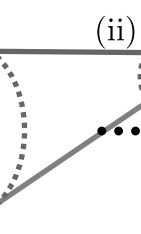

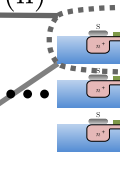
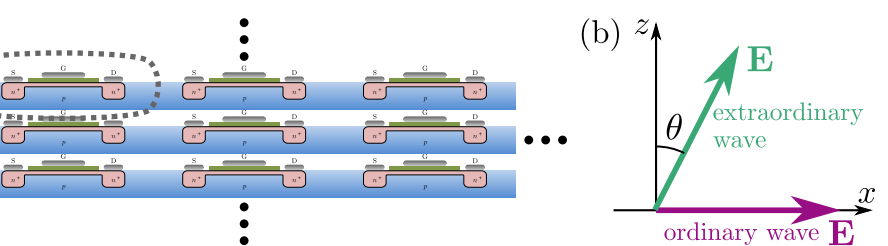

FIG. 1. (a)(i) Sketch of a MOSFET transistor. (ii) Illustration of a metamaterial formed by a periodic array of MOSFETs. (b) Geometrical relation between the E-fields of the plane wave modes in the MOSFET-metamaterial [Eq. (5)] for propagation along $y$.

formed by many structural unities identical to the MOSFET organized in a lattice (Fig. 1(a)-(ii)). Hereafter we refer to this medium as the MOSFET-metamaterial. We note that metamaterials loaded with transistors have been discussed in seminal works by Caloz and 
others [51 53] in a different context.

The material constitutive relation that mimics the response of a MOSFET transistor is of the form $\mathbf{P}=\varepsilon_{0} \bar{\chi}(\mathbf{E}) \cdot \mathbf{E}$ with

$$
\bar{\chi}(\mathbf{E})=\left(\begin{array}{ccc}
\chi_{x x}\left(E_{z}\right) & 0 & 0 \\
0 & \chi_{y y} & 0 \\
0 & 0 & \chi_{z z}
\end{array}\right)
$$

Here, $\mathbf{P}$ is the polarization vector in the metamaterial and $\mathbf{E}$ is the electric field. The susceptibility $\chi_{x x}\left(E_{z}\right)$ depends on the field strength along $z$, in the same manner as the MOSFET impedance along the drain-to-source channel (x-direction) depends on the gate voltage $(z$ component of the electric field). Thus, the metamaterial is nonlinear. The polarizabilities $\chi_{y y}$ and $\chi_{z z}$ are assumed independent of the field strength. For simplicity we neglect material dispersion so that the susceptibility is frequency independent and real-valued. The dispersive effects could be modelled by noting that the material response is determined by a set of nonlinear differential equations, which could be linearized using a procedure analogous to what is described below. The main impact of material dispersion is that it usually determines a frequency cutoff beyond which the nonlinear response becomes too weak, and, in addition, the permittivity components may become complex-valued.

Let us suppose that such hypothetical metamaterial is biased with some static electric field in the $x o z$ plane $\mathbf{E}_{0}=E_{0 x} \hat{\mathbf{x}}+E_{0 z} \hat{\mathbf{z}}$. For small field variations $(\delta \mathbf{E})$ around the biasing point $\left(\mathbf{E}=\mathbf{E}_{0}+\delta \mathbf{E}\right)$ the electric response can be linearized as,

$$
\mathbf{P} \approx \varepsilon_{0} \bar{\chi}\left(\mathbf{E}_{0}\right) \cdot \mathbf{E}_{0}+\left.\varepsilon_{0} \sum_{i=x, y, z} \partial_{E_{i}}[\bar{\chi}(\mathbf{E}) \cdot \mathbf{E}]\right|_{\mathbf{E}=\mathbf{E}_{0}} \delta E_{i}
$$

In the above, $\partial_{E_{i}} \equiv \partial / \partial E_{i}$ with $i=x, y, z$, represents a derivative with respect to the electric field. The induced polarization is $\mathbf{P}=\mathbf{P}_{0}+\delta \mathbf{P}$, with $\delta \mathbf{P}=\left.\varepsilon_{0} \sum_{i=x, y, z} \partial_{E_{i}}[\bar{\chi}(\mathbf{E}) \cdot \mathbf{E}]\right|_{\mathbf{E}=\mathbf{E}_{0}} \delta E_{i}$ the linearized signal response. Thus, for sufficiently weak signals, the dynamical parts of the polarization and electric field vectors are related through a linear relation of the form $\delta \mathbf{P}=\varepsilon_{0} \bar{\chi}_{\text {lin }} \cdot \delta \mathbf{E}$ where $\bar{\chi}_{\text {lin }}$ is the effective (linearized) material susceptibility. It can be written explicitly as:

$$
\bar{\chi}_{\text {lin }}=\left.\sum_{i=x, y, z} \partial_{E_{i}}[\bar{\chi}(\mathbf{E}) \cdot \mathbf{E}]\right|_{\mathbf{E}=\mathbf{E}_{0}} \otimes \hat{\mathbf{u}}_{i}
$$


with $\hat{\mathbf{u}}_{i}$ a unit vector directed along the $i$-th direction, and $\otimes$ represents the tensor product of two vectors. For the particular model in Eq. (11), one finds that

$$
\bar{\chi}_{\text {lin }}=\left(\begin{array}{ccc}
\chi_{x x}\left(E_{0 z}\right) & 0 & \left.\partial_{E_{z}} \chi_{x x}\right|_{\mathbf{E}=\mathbf{E}_{0}} E_{0 x} \\
0 & \chi_{y y} & 0 \\
0 & 0 & \chi_{z z}
\end{array}\right) .
$$

In general, the linearized response is nonreciprocal because $\bar{\chi}_{\text {lin }} \neq \bar{\chi}_{\text {lin }}^{T}$ (the superscript $T$ represents the matrix transpose). Thus, our proposal establishes a novel paradigm to have nonreciprocity with an electric bias. A nonreciprocal response requires that the bias static field has an $x$-component $\left(E_{0 x} \neq 0\right)$, i.e. a component along the drain-to-source direction in the MOSFET transistor analogue. The field component $E_{0 z}$ may be zero when $\left.\partial_{E_{z}} \chi_{x x}\right|_{E_{z}=0} \neq 0$.

It is rather curious that the microscopic susceptibility is a symmetric tensor (Eq.(1)), but the linearized susceptibility (Eq.(4)) is not. Furthermore, for the considered model, the linearization preserves the TR symmetry of the system. We note in passing that Ref. [54] studied related (but not equivalent) non-Hermitian material responses obtained using optical pumping. In general, a non-Hermitian material response must be rooted in some nonequilibrium process that can extract energy from either the stored electrostatic field or from the DC generator. The latter case is feasible with a drift current, analogous to a MOSFET transistor. Note that in our scheme the drift velocity can be a tiny fraction of the wave velocity, different from other solutions studied previously that rely on materials with large mobility [39-47].

Even though our starting point was an analogy with transistors, related nonreciprocal responses can in principle be engineered using naturally available nonlinear materials. For example, related nonlinearities may naturally occur in crystals without inversion symmetry [86] and may also be engineered in semiconductor superlattices [87]. It is however essential that the material is operated in nonequilibrium (e.g., with carrier injection), as in equilibrium the Kleinman symmetry forces the linearized response to be reciprocal [88, 89].

Since the electric displacement vector is $\mathbf{D}=\varepsilon_{0} \mathbf{E}+\mathbf{P}$, the linearized relative permittivity of the metamaterial is $\bar{\varepsilon}=\mathbf{1}_{3 \times 3}+\bar{\chi}_{\text {lin }}$. Thus, for a MOSFET-metamaterial the permittivity 
tensor is of the form:

$$
\bar{\varepsilon}=\left(\begin{array}{ccc}
\varepsilon_{x x} & 0 & \varepsilon_{x z} \\
0 & \varepsilon_{y y} & 0 \\
0 & 0 & \varepsilon_{z z}
\end{array}\right)
$$

This formula confirms that when the permittivity tensor is real-valued and frequency independent, the linearized response remains TR invariant $\left(\bar{\varepsilon}^{*}=\bar{\varepsilon}\right)$, even though it is nonreciprocal $\left(\bar{\varepsilon}^{T} \neq \bar{\varepsilon}\right)[90]$. Furthermore, the metamaterial is non-Hermitian $\left(\bar{\varepsilon}^{\dagger} \neq \bar{\varepsilon}\right)$, which indicates that it can either absorb or generate energy.

For simplicity, in the remainder of this Letter, we focus on the material response of the idealized MOSFET-metamaterial (5). We are interested only in weak signals described by the linearized response, and thus hereafter, to keep the notations short, we drop the $\delta$ symbol $(\delta \mathbf{E} \rightarrow \mathbf{E})$, and denote the dynamic electric field simply by $\mathbf{E}$.

Next, we characterize the plane wave modes in the metamaterial. The wave equation is of the form $\nabla \times \nabla \times \mathbf{E}=\frac{\omega^{2}}{c^{2}} \bar{\varepsilon} \cdot \mathbf{E}$, where $\omega$ is the angular frequency and $c$ the speed of light in vacuum. Plane wave solutions are of the form $\mathbf{E}=\mathbf{A}_{0} \mathrm{e}^{i \mathbf{k} \cdot \mathbf{r}}$ with $\mathbf{A}_{0}$ a constant complex vector, $\mathbf{k}$ the wave vector and $\mathbf{r}=x \hat{\mathbf{x}}+y \hat{\mathbf{y}}+z \hat{\mathbf{z}}$ the position vector. We restrict our attention to propagation along the $y$ - direction, i.e., the direction perpendicular to the Gate-Drain-Source plane (see Fig. 1(a)), so that $\mathbf{k}=k \hat{\mathbf{y}}$. In this case, the solutions of the homogeneous wave equation are such that:

$$
\begin{aligned}
\mathbf{k}=\frac{\omega}{c} \sqrt{\varepsilon_{x x}} \hat{\mathbf{y}} \equiv \mathbf{k}_{o}, & \mathbf{E} \sim \hat{\mathbf{x}} & \text { (ordinary wave) } \\
\mathbf{k}=\frac{\omega}{c} \sqrt{\varepsilon_{z z}} \hat{\mathbf{y}} \equiv \mathbf{k}_{e}, & \mathbf{E} \sim \frac{\varepsilon_{x z}}{\varepsilon_{z z}-\varepsilon_{x x}} \hat{\mathbf{x}}+\hat{\mathbf{z}} & \text { (extraordinary wave) }
\end{aligned}
$$

Remarkably, due to the non-Hermitian response for a non-zero cross-coupling coefficient $\varepsilon_{x z}$, the ordinary and extraordinary waves are not orthogonal [54, 91]. The tilt of the extraordinary wave electric field with respect to the direction perpendicular to the ordinary wave is determined by an angle $\theta$ such that $\tan (\theta)=\frac{\varepsilon_{x z}}{\varepsilon_{z z}-\varepsilon_{x x}}$ (see Fig. 1(b)). Interestingly, $\theta$ determines the strength of the nonreciprocal effects. It may differ significantly from zero even for a small cross-coupling coefficient $\varepsilon_{x z}$ provided the values of $\varepsilon_{x x}$ and $\varepsilon_{z z}$ are sufficiently close. The limiting case $\varepsilon_{x x}=\varepsilon_{z z}$ corresponds to an exceptional point where the two waves (6) coalesce into a single linear polarization [54] and will not be further considered here. 
Consider a superposition of ordinary and extraordinary waves propagating along $+y$ :

$$
\mathbf{E}=A_{o} \mathrm{e}^{i k_{o} y} \hat{\mathbf{x}}+A_{e}\left(\frac{\varepsilon_{x z}}{\varepsilon_{z z}-\varepsilon_{x x}} \hat{\mathbf{x}}+\hat{\mathbf{z}}\right) \mathrm{e}^{i k_{e} y}
$$

The corresponding magnetic field is given by $\mathbf{H}=\frac{-i}{\omega \mu_{0}} \hat{\mathbf{y}} \times \partial_{y} \mathbf{E}$ (with $\partial_{y} \equiv \partial / \partial y$ ). After some algebra, one may show that the time-averaged Poynting vector $\mathbf{S}=\frac{1}{2} \operatorname{Re}\left\{\mathbf{E} \times \mathbf{H}^{*}\right\}$ in the material is:

$\mathbf{S}=\frac{1}{2} \frac{\sqrt{\varepsilon_{0}}}{\sqrt{\mu_{0}}}\left(\left|A_{o}\right|^{2} \sqrt{\varepsilon_{x x}}+\left|A_{e}\right|^{2} \sqrt{\varepsilon_{z z}}\left(1+\left|\frac{\varepsilon_{x z}}{\varepsilon_{z z}-\varepsilon_{x x}}\right|^{2}\right)+\frac{1}{\sqrt{\varepsilon_{z z}}-\sqrt{\varepsilon_{x x}}} \operatorname{Re}\left\{\varepsilon_{x z} A_{e} A_{o}^{*} \mathrm{e}^{i\left(k_{e}-k_{o}\right) y}\right\}\right) \hat{\mathbf{y}}$

The first two terms of this expression represent the power transported by the ordinary and the extraordinary waves alone, respectively. Remarkably, provided the cross-coupling coefficient $\varepsilon_{x z}$ does not vanish, there is a third term that is responsible for a periodic spatial variation of the power flux. This third term describes a power beating with spatial frequency $k_{e}-k_{o}$ as illustrated with a numerical example in Fig. 2. Thus, when $\varepsilon_{x z} \neq 0$, the two modes

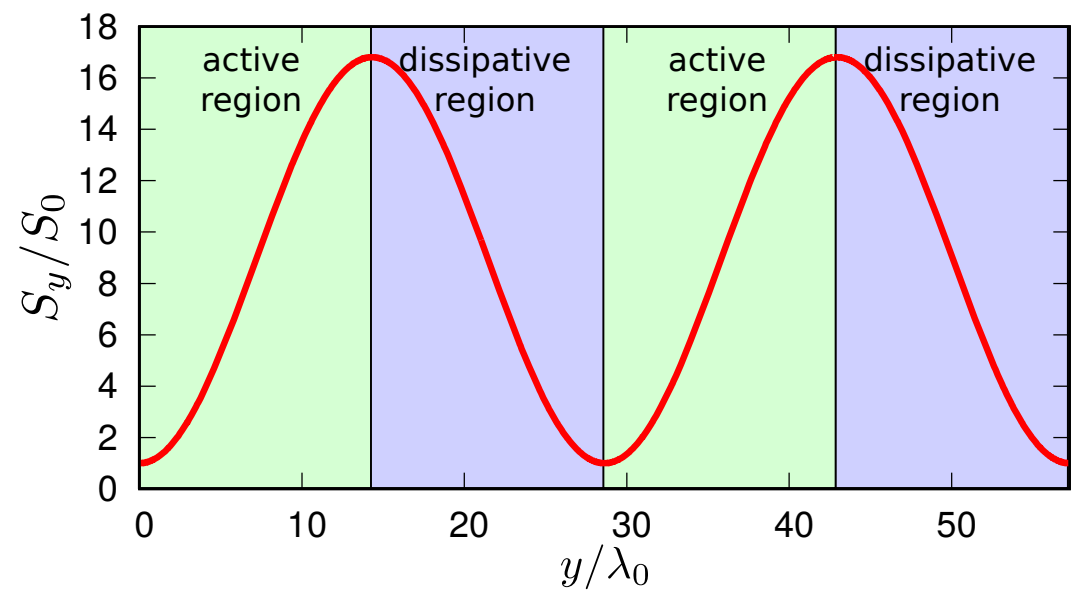

FIG. 2. Normalized Poynting vector as a function of the propagation distance $y$ normalized to the vacuum wavelength $\lambda_{0}=2 \pi c / \omega$. The parameters are $\varepsilon_{x x}=2, \varepsilon_{z z}=2.1, \varepsilon_{x z}=0.2, A_{e}=1$ and $A_{o}=-A_{e} \frac{\varepsilon_{x z}}{\varepsilon_{z z}-\varepsilon_{x x}}$. The regions shaded in green correspond to active regions, whereas the regions shaded in purple correspond to dissipative regions.

cease to transport the power independently in the material. This is a rather unique result, as for any Hermitian system the electromagnetic modes always transport power independently. In fact, if the crossed term of the Poynting vector does not vanish, the power flux forcibly 
depends on the propagation distance (as in Fig. 22). This is only possible in non-energy conserving (non-Hermitian) systems, e.g., systems with loss or gain $\left(\bar{\varepsilon} \neq \bar{\varepsilon}^{\dagger}\right)$.

Curiously, the interplay of the static bias field with the material nonlinearity may lead to either dissipation or gain. Thus, our metamaterial may behave as either "lossy" or "gainy", depending on the relative phase between the two propagating modes. In different words, the material is neither a standard lossy material nor a standard gainy material, but rather exhibits a dual-type response. In particular, depending on the relative phase between the ordinary and extraordinary waves, the MOSFET-metamaterial alternates between active and dissipative regions (see the supplementary information [92]). For the particular model considered here, the energy exchange between the wave and the medium is controlled by a polarization current induced by the static electric field through the nonlinearity [92]. The power amplification is proportional to the magnitude of the cross-coupling coefficient and can be made rather large if the values of $\varepsilon_{x x}$ and $\varepsilon_{z z}$ are sufficiently close. Similar power beatings may occur in other non-Hermitian platforms (e.g., [54]).

Next, we present a design of an electromagnetic isolator. The proposed system is depicted in Fig. 3(a) and is based on the non-orthogonality of the two eigenvectors of the bulk material. It consists of a MOSFET-metamaterial slab of thickness $d$ placed in between two orthogonal linear polarizers. The linear polarizers are supposed to fully absorb the electric field component parallel to some axis and let the orthogonal component pass through them unchanged.

We introduce the transmission matrix $\bar{T}$ that relates (in the absence of the polarizing grids) the transverse components of the incident $\mathbf{E}_{\mathrm{t}}^{\mathrm{inc}}=\left(E_{x}^{\mathrm{inc}} E_{z}^{\mathrm{inc}}\right)^{T}$ and transmitted $\mathbf{E}_{\mathrm{t}}^{\mathrm{tr}}=$ $\left(\begin{array}{ll}E_{x}^{\operatorname{tr}} & E_{z}^{\operatorname{tr}}\end{array}\right)^{T}$ electric fields as $\mathbf{E}_{\mathrm{t}}^{\mathrm{tr}}=\bar{T} \cdot \mathbf{E}_{\mathrm{t}}^{\mathrm{inc}}$. In the supplementary information [92], it is shown that for normal incidence $(\mathbf{k}=k \hat{\mathbf{y}}$ with $k=\omega / c)$ the transmission matrix for a material slab with thickness $d$ is

$$
\bar{T}=\left(\begin{array}{cc}
\gamma_{o} & \frac{\varepsilon_{x z}}{\varepsilon_{x x}-\varepsilon_{z z}}\left(\gamma_{o}-\gamma_{e}\right) \\
0 & \gamma_{e}
\end{array}\right)
$$

where $\gamma_{j}=\left(\cos \left(k_{j} d\right)-\frac{i\left(k_{j}^{2}+k^{2}\right)}{2 k_{j} k} \sin \left(k_{j} d\right)\right)^{-1}$ with $j=o$, e. The transmission matrix lacks transpose symmetry and is independent of the direction of propagation of the incoming wave. The matrix has a single nonzero anti-diagonal element, and hence the slab can generate an outgoing wave with $E_{x}$ component from an incoming wave polarized along $z$, but not the 

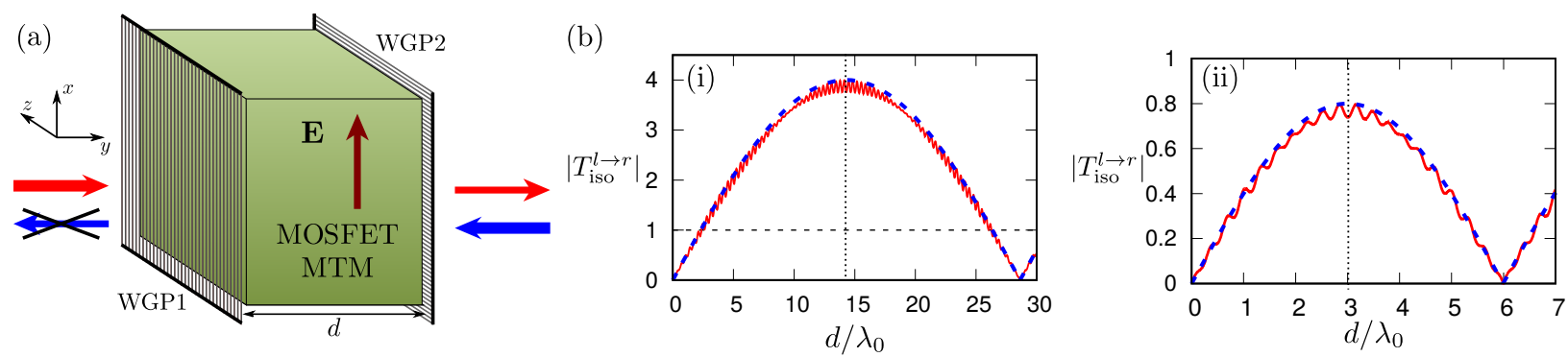

FIG. 3. (a) Electromagnetic isolator based on an electrically biased MOSFET-metamaterial placed in between two orthogonal linear polarizers. To ease the vizualization the polarizers are represented as wire grids, WGP1 and WGP2, that absorb electric fields parallel to the directions of the wires, i.e., the $x$ and $z$ directions, respectively. The propagation from right-to-left is forbidden. The direction of the electric bias $\mathbf{E}_{0}$ is also shown. (b) Transmission from left to right $\left|T_{\text {iso }}^{l \rightarrow r}\right|$ as a function of the isolator thickness $d$ normalized to the free-space wavelength. The parameters are $\varepsilon_{x x}=2, \varepsilon_{x z}=0.2$ and (i) $\varepsilon_{z z}=2.1$ and (ii) $\varepsilon_{z z}=2.5$. The red line is the exact solution whereas the blue dashed line is the approximate solution given by Eq.(10). The vertical dotted lines mark the positions of $d_{\max }$.

\section{converse.}

Let us now analyze the response of the proposed isolator. For an incident wave propagating from left-to-right the polarizing grid WPG1 ensures that the field that reaches the metamaterial is oriented along the $z$ direction with an amplitude $E_{z}^{\text {inc }}$. Due to the cross-coupling term $\varepsilon_{x z}$, the propagation in the metamaterial may induce an electric field component along the $x$-direction. This $x$-component of the field can go through the grid WPG2, generating the left to right output signal. In contrast, an incident wave propagating from right-to-left can enter into the MOSFET-metamaterial only if the incident electric field has a component along $x$. In this case, the metamaterial does not generate any cross-polarization component, and thereby the incoming wave is fully absorbed at the output polarizing grid. Therefore, the wave propagation is allowed only in a specific (left-to-right) direction.

This is confirmed by Eq.(9) that reveals that the right to left transmission coefficient $T_{\text {iso }}^{r \rightarrow l}=T_{21}$ of the isolator is exactly zero. The left-to-right transmission $\left|T_{\text {iso }}^{l \rightarrow r}\right|=T_{12}$ is depicted in Fig. 3(b) as a function of the isolator thickness $d$ for different material parameters. As seen, a longer propagation distance typically yields a stronger output signal, up to some threshold value beyond which an oscillatory behavior is observed. By neglecting the 
reflections at the air-metamaterial interfaces, one finds that [92]:

$$
\left|T_{\text {iso }}^{l \rightarrow r}\right| \approx\left|2 \frac{\varepsilon_{x z}}{\varepsilon_{z z}-\varepsilon_{x x}} \sin \left(\frac{k_{e}-k_{o}}{2} d\right)\right| .
$$

Hence, the transmission is maximized when $\frac{k_{e}-k_{o}}{2} d=\frac{\pi}{2}$, i.e. for a thickness $d=d_{\max } \equiv$ $\frac{\lambda_{0}}{2\left(\sqrt{\varepsilon_{z z}}-\sqrt{\varepsilon_{x x}}\right)}$. The transmission maximum is $|2 \tan (\theta)|$ and is controlled by the angle $\theta$ and by the non-orthogonality of the eigenvectors (see Fig. 1(b)). The transmission level can in principle be much greater than unity due to the active response of the metamaterial (see Fig. 3 (b)-(i)).

For a fixed value of $\varepsilon_{x z}$, a larger detuning of the diagonal elements $\left|\varepsilon_{x x}-\varepsilon_{z z}\right|$ leads to a decrease in $d_{\max }$, but at the same time the transmission level drops down. This is illustrated in Fig. 3(b) where the strong amplification obtained in (i) after a propagation of around $15 \lambda_{0}$ becomes smaller than unity but the maximum is reached only after $3 \lambda_{0}$.

For a device with small $d$ Eq. (10) becomes $\left|T_{\text {iso }}^{l \rightarrow r}\right| \approx \frac{2 \pi d}{\lambda_{0}}\left|\frac{\varepsilon_{x z}}{\sqrt{\varepsilon_{z z}}+\sqrt{\varepsilon_{x x}}}\right|$. Hence, for a small thickness the amplification factor is bounded by the amplitude of $\varepsilon_{x z}$. In contrast, for a large thickness this limitation disappears, because the distributed gain is controlled by $\frac{\varepsilon_{x z}}{\varepsilon_{z z}-\varepsilon_{x x}}$, and thus can be arbitrarily large.

It is relevant to point out that even though the idealized metamaterial is TR invariant, the presence of the dissipative polarizing grids breaks the TR symmetry of the system (Fig. [3(a)). In fact, it is well known that dissipation is an absolutely essential ingredient to realize an electromagnetic isolator. Moreover, our system is not affected by any of the problems that limit the performance of systems that exploit dynamic nonlinearities [77-79]. In principle our isolator can be implemented using any linearized material response with non-orthogonal eigenvectors.

In summary, we introduced a new and robust mechanism to break the Lorentz reciprocity using the combination of a static electric bias and material nonlinearities. Starting from an analogy with the operation of a semiconductor transistor, we showed that the linearization of a nonlinear material response, can lead to a non-symmetric and non-Hermitian permittivity response. We studied in detail the wave propagation in an idealized MOSFET-metamaterial, showing that the non-Hermitian nature of the wave-matter interactions opens many exciting opportunities and can lead to a dual material behavior, where regimes of absorption alternate with regimes of gain in the same physical system. Furthermore, we suggested a design of an electromagnetic isolator using the proposed material, whose performance may in principle 
surpass that of standard Faraday isolators. We envision that related platforms can be engineered as metamaterials or, alternatively, can be implemented using naturally available materials in nonequilibrium situations. The prospects are especially promising up to $\mathrm{THz}$ frequencies, both in metamaterial [93] and in natural material realizations. Note that the nonlinear response of naturally available materials remains strong and fast enough up to the longitudinal optical phonon resonance [94].

\section{ACKNOWLEDGMENTS}

This work was partially funded by Huawei Technologies, by the Institution of Engineering and Technology (IET) under the A F Harvey Research Prize 2018, by the Simons Foundation under the award 733700 (Simons Collaboration in Mathematics and Physics, "Harnessing Universal Symmetry Concepts for Extreme Wave Phenomena") and by Instituto de Telecomunicações under Project Nº. UID/EEA/50008/2020. S.L. acknowledges FCT and IT-Coimbra for the research financial support with reference DL 57/2016/CP1353/CT000. D.E.F. acknowledges support by FCT, POCH, and the cofinancing of Fundo Social Europeu under the fellowship SFRH/BPD/116525/2016. T. A. M. acknowledges FCT for research financial support with reference CEECIND/04530/2017 under the CEEC Individual 2017, and IT-Coimbra for the contract as an assistant researcher with reference CT/Nº 004/2019F00069.

\section{REFERENCES}

* To whom correspondence should be addressed: mario.silveirinha@co.it.pt

[1] D. M. Pozar, Microwave Engineering, 4th ed. (John Wiley \& Sons, Hoboken, NJ, 2011).

[2] C. Caloz, A. Alù, S. Tretyakov, D. Sounas, K. Achouri, and Z.-L. Deck-Léger, "Electromagnetic Nonreciprocity," Phys. Rev. Applied 10, 047001 (2018).

[3] M. G. Silveirinha, "Time-reversal Symmetry in Antenna Theory," Symmetry 11, 486 (2019).

[4] J. D. Adam, L. E. Davis, G. F. Dionne, E. F. Schloemann, and S. N. Stitzer, "Ferrite devices and materials," 
IEEE Transactions on Microwave Theory and Techniques 50, 721-737 (2002).

[5] N. A. Krall and A. W. Trivelpiece, Principles of Plasma Physics (McGraw-Hill, 1973).

[6] L. P. Pitaevskii and E. M. Lifshitz, Physical Kinetics: Volume 10, 1st ed. (ButterworthHeinemann, Amsterdam, 1981).

[7] H. Dötsch, N. Bahlmann, O. Zhuromskyy, M. Hammer, L. Wilkens, R. Gerhardt, P. Hertel, and A. F. Popkov, "Applications of magneto-optical waveguides in integrated optics: review," J. Opt. Soc. Am. B, JOSAB 22, 240-253 (2005).

[8] M. G. Silveirinha, "Chern invariants for continuous media," Phys. Rev. B 92, 125153 (2015).

[9] F. D. M. Haldane and S. Raghu, "Possible Realization of Directional Optical Waveguides in Photonic Crystals with Broken Time-Reversal Symmetry," Physical Review Letters 100, 013904 (2008).

[10] S. Raghu and F. D. M. Haldane, "Analogs of quantum-Hall-effect edge states in photonic crystals," Physical Review A 78, 033834 (2008).

[11] Z. Yu, G. Veronis, Z. Wang, and S. Fan, "One-Way Electromagnetic Waveguide Formed at the Interface between a Plasmonic Metal under a Static Magnetic Field and a Photonic Crystal," Phys. Rev. Lett. 100, 023902 (2008).

[12] Z. Wang, Y. Chong, J. D. Joannopoulos, and M. Soljačić, "Observation of unidirectional backscattering-immune topological electromagnetic states," Nature 461, 772-775 (2009).

[13] A. R. Davoyan and N. Engheta, "Theory of Wave Propagation in Magnetized Near-ZeroEpsilon Metamaterials: Evidence for One-Way Photonic States and Magnetically Switched Transparency and Opacity," Phys. Rev. Lett. 111, 257401 (2013).

[14] X. Lin, Y. Xu, B. Zhang, R. Hao, H. Chen, and E. Li, "Unidirectional surface plasmons in nonreciprocal graphene," New J. Phys. 15, 113003 (2013).

[15] L. Lu, J. D. Joannopoulos, and M. Soljačić, "Topological photonics," Nature Photonics 8, 821-829 (2014).

[16] S. A. H. Gangaraj and G. W. Hanson, "Topologically Protected Unidirectional Surface States in Biased Ferrites: Duality and Application to Directional Couplers," IEEE Antennas and Wireless Propagation Letters 16, 449-452 (2017).

[17] A. B. Khanikaev and G. Shvets, "Two-dimensional topological photonics," Nature Photonics 11, 763-773 (2017). 
[18] D. E. Fernandes and M. G. Silveirinha, "Topological Origin of Electromagnetic Energy Sinks," Phys. Rev. Applied 12, 014021 (2019).

[19] S. Lannebère and M. G. Silveirinha, "Link between the photonic and electronic topological phases in artificial graphene," Phys. Rev. B 97, 165128 (2018).

[20] S. Lannebère and M. G. Silveirinha, "Photonic analogues of the Haldane and Kane-Mele models," Nanophotonics 8, 1387-1397 (2019).

[21] Z. Yu and S. Fan, "Complete optical isolation created by indirect interband photonic transitions," Nature Photonics 3, 91-94 (2009).

[22] S. Manipatruni, J. T. Robinson, and M. Lipson, "Optical Nonreciprocity in Optomechanical Structures," Phys. Rev. Lett. 102, 213903 (2009).

[23] A. Kamal, J. Clarke, and M. H. Devoret, "Noiseless non-reciprocity in a parametric active device," Nature Physics 7, 311-315 (2011).

[24] X. Huang and S. Fan, "Complete All-Optical Silica Fiber Isolator via Stimulated Brillouin Scattering," Journal of Lightwave Technology 29, 2267-2275 (2011).

[25] K. Fang, Z. Yu, and S. Fan, "Photonic Aharonov-Bohm Effect Based on Dynamic Modulation," Phys. Rev. Lett. 108, 153901 (2012).

[26] D. L. Sounas, C. Caloz, and A. Alù, "Giant non-reciprocity at the subwavelength scale using angular momentum-biased metamaterials," Nature Communications 4, 2407 (2013).

[27] D.-W. Wang, H.-T. Zhou, M.-J. Guo, J.-X. Zhang, J. Evers, and S.-Y. Zhu, "Optical Diode Made from a Moving Photonic Crystal," Phys. Rev. Lett. 110, 093901 (2013).

[28] S. Hua, J. Wen, X. Jiang, Q. Hua, L. Jiang, and M. Xiao, "Demonstration of a chip-based optical isolator with parametric amplification," Nature Communications 7, 13657 (2016).

[29] D. L. Sounas and A. Alù, "Non-reciprocal photonics based on time modulation," Nature Photonics 11, 774-783 (2017).

[30] F. Ruesink, M.-A. Miri, A. Alù, and E. Verhagen, "Nonreciprocity and magnetic-free isolation based on optomechanical interactions," Nature Communications 7, 13662 (2016).

[31] M.-A. Miri, F. Ruesink, E. Verhagen, and A. Alù, "Optical Nonreciprocity Based on Optomechanical Coupling," Phys. Rev. Applied 7, 064014 (2017).

[32] F. Ruesink, J. P. Mathew, M.-A. Miri, A. Alù, and E. Verhagen, "Optical circulation in a multimode optomechanical resonator," Nature Communications 9, 1798 (2018). 
[33] E. Galiffi, P. A. Huidobro, and J. B. Pendry, "Broadband Nonreciprocal Amplification in Luminal Metamaterials," Phys. Rev. Lett. 123, 206101 (2019).

[34] A. Mock, D. Sounas, and A. Alù, "Magnet-Free Circulator Based on Spatiotemporal Modulation of Photonic Crystal Defect Cavities," ACS Photonics 6, 2056-2066 (2019).

[35] R. Duggan, D. Sounas, and A. Alu, "Optically driven effective Faraday effect in instantaneous nonlinear media," Optica, OPTICA 6, 1152-1157 (2019).

[36] S. A. R. Horsley, J.-H. Wu, M. Artoni, and G. C. La Rocca, "Optical Nonreciprocity of Cold Atom Bragg Mirrors in Motion," Phys. Rev. Lett. 110, 223602 (2013).

[37] R. Fleury, D. L. Sounas, C. F. Sieck, M. R. Haberman, and A. Alù, "Sound Isolation and Giant Linear Nonreciprocity in a Compact Acoustic Circulator," Science 343, 516-519 (2014).

[38] S. Lannebère and M. G. Silveirinha, "Wave instabilities and unidirectional light flow in a cavity with rotating walls," Phys. Rev. A 94, 033810 (2016).

[39] D. S. Borgnia, T. V. Phan, and L. S. Levitov, "Quasi-Relativistic Doppler Effect and NonReciprocal Plasmons in Graphene," arXiv:1512.09044 [cond-mat] (2015).

[40] B. V. Duppen, A. Tomadin, A. N. Grigorenko, and M. Polini, "Current-induced birefringent absorption and non-reciprocal plasmons in graphene," 3, 015011 (2016).

[41] T. A. Morgado and M. G. Silveirinha, "Drift-Induced Unidirectional Graphene Plasmons," ACS Photonics 5, 4253-4258 (2018).

[42] T. Wenger, G. Viola, J. Kinaret, M. Fogelström, and P. Tassin, "Current-controlled light scattering and asymmetric plasmon propagation in graphene," Phys. Rev. B 97, 085419 (2018).

[43] K. Y. Bliokh, F. J. Rodríguez-Fortuño, A. Y. Bekshaev, Y. S. Kivshar, and F. Nori, "Electric-current-induced unidirectional propagation of surface plasmon-polaritons," Opt. Lett., OL 43, 963-966 (2018).

[44] T. A. Morgado and M. G. Silveirinha, "Nonlocal effects and enhanced nonreciprocity in current-driven graphene systems," Phys. Rev. B 102, 075102 (2020).

[45] T. A. Morgado and M. G. Silveirinha, "Active Graphene Plasmonics with a Drift-Current Bias," ACS Photonics 8, 1129-1136 (2021).

[46] W. Zhao, S. Zhao, H. Li, S. Wang, S. Wang, M. Iqbal Bakti Utama, S. Kahn, Y. Jiang, X. Xiao, S.J. Yoo, K. Watanabe, T. Taniguchi, A. Zettl, and F. Wang, "Efficient Fizeau drag from Dirac electrons in monolayer graphene," Nature 594, 517-521 (2021). 
[47] Y. Dong, L. Xiong, I. Y. Phinney, Z. Sun, R. Jing, A. S. McLeod, S. Zhang, S. Liu, F. L. Ruta, H. Gao, Z. Dong, R. Pan, J. H. Edgar, P. Jarillo-Herrero, L. S. Levitov, A. J. Millis, M. M. Fogler, D. A. Bandurin, and D. N. Basov, "Fizeau drag in graphene plasmonics," Nature 594, 513-516 (2021).

[48] K. G. Makris, R. El-Ganainy, D. N. Christodoulides, and Z. H. Musslimani, "Beam Dynamics in $\mathcal{P} \mathcal{T}$ Symmetric Optical Lattices," Phys. Rev. Lett. 100, 103904 (2008).

[49] C. E. Rüter, K. G. Makris, R. El-Ganainy, D. N. Christodoulides, M. Segev, and D. Kip, "Observation of parity-time symmetry in optics," Nature Physics 6, 192-195 (2010).

[50] Z. Lin, J. Schindler, F. M. Ellis, and T. Kottos, "Experimental observation of the dual behavior of $\mathcal{P} \mathcal{T}$-symmetric scattering," Phys. Rev. A 85, 050101(R) (2012).

[51] T. Kodera, D. L. Sounas, and C. Caloz, "Artificial Faraday rotation using a ring metamaterial structure without static magnetic field," Applied Physics Letters 99, 031114 (2011).

[52] Z. Wang, Z. Wang, J. Wang, B. Zhang, J. Huangfu, J. D. Joannopoulos, M. Soljačić, and L. Ran, "Gyrotropic response in the absence of a bias field," PNAS 109, 13194-13197 (2012).

[53] T. Kodera, D. L. Sounas, and C. Caloz, "Magnetless Nonreciprocal Metamaterial (MNM) Technology: Application to Microwave Components," IEEE Transactions on Microwave Theory and Techniques 61, 1030-1042 (2013).

[54] S. Buddhiraju, A. Song, G. T. Papadakis, and S. Fan, "Nonreciprocal Metamaterial Obeying Time-Reversal Symmetry," Phys. Rev. Lett. 124, 257403 (2020).

[55] M. Scalora, J. P. Dowling, C. M. Bowden, and M. J. Bloemer, "The photonic band edge optical diode," Journal of Applied Physics 76, 2023-2026 (1994).

[56] M. D. Tocci, M. J. Bloemer, M. Scalora, J. P. Dowling, and C. M. Bowden, "Thin-film nonlinear optical diode," Appl. Phys. Lett. 66, 2324-2326 (1995).

[57] K. Gallo and G. Assanto, "All-optical diode based on second-harmonic generation in an asymmetric waveguide," J. Opt. Soc. Am. B, JOSAB 16, 267-269 (1999).

[58] K. Gallo, G. Assanto, K. R. Parameswaran, and M. M. Fejer, "All-optical diode in a periodically poled lithium niobate waveguide," Appl. Phys. Lett. 79, 314-316 (2001).

[59] S. F. Mingaleev and Y. S. Kivshar, "Nonlinear transmission and light localization in photoniccrystal waveguides," J. Opt. Soc. Am. B, JOSAB 19, 2241-2249 (2002).

[60] H. Zhou, K.-F. Zhou, W. Hu, Q. Guo, S. Lan, X.-S. Lin, and A. Venu Gopal, "Alloptical diodes based on photonic crystal molecules consisting of nonlinear defect pairs," 
Journal of Applied Physics 99, 123111 (2006).

[61] X.-S. Lin, J.-H. Yan, L.-J. Wu, and S. Lan, "High transmission contrast for single resonator based all-optical diodes with pump-assisting," Opt. Express, OE 16, 20949-20954 (2008).

[62] A. E. Miroshnichenko, E. Brasselet, and Y. S. Kivshar, "Reversible optical nonreciprocity in periodic structures with liquid crystals," Appl. Phys. Lett. 96, 063302 (2010).

[63] I. V. Shadrivov, V. A. Fedotov, D. A. Powell, Y. S. Kivshar, and N. I. Zheludev, "Electromagnetic wave analogue of an electronic diode," New J. Phys. 13, 033025 (2011).

[64] S. Lepri and G. Casati, "Asymmetric Wave Propagation in Nonlinear Systems," Phys. Rev. Lett. 106, 164101 (2011).

[65] S. V. Zhukovsky and A. G. Smirnov, "All-optical diode action in asymmetric nonlinear photonic multilayers with perfect transmission resonances," Phys. Rev. A 83, 023818 (2011).

[66] W. Ding, B. Luk'yanchuk, and C.-W. Qiu, "Ultrahigh-contrast-ratio silicon Fano diode," Phys. Rev. A 85, 025806 (2012).

[67] Y. Xu and A. E. Miroshnichenko, "Reconfigurable nonreciprocity with a nonlinear Fano diode," Phys. Rev. B 89, 134306 (2014).

[68] Y. Yu, Y. Chen, H. Hu, W. Xue, K. Yvind, and J. Mork, "Nonreciprocal transmission in a nonlinear photonic-crystal Fano structure with broken symmetry," Laser \& Photonics Reviews 9, 241-247 (2015).

[69] D. L. Sounas, J. Soric, and A. Alù, "Broadband passive isolators based on coupled nonlinear resonances," Nature Electronics 1, 113-119 (2018).

[70] A. A. Sukhorukov, Z. Xu, and Y. S. Kivshar, "Nonlinear suppression of time reversals in $\mathcal{P} \mathcal{T}$-symmetric optical couplers," Phys. Rev. A 82, 043818 (2010).

[71] H. Ramezani, T. Kottos, R. El-Ganainy, and D. N. Christodoulides, "Unidirectional nonlinear $\mathcal{P} \mathcal{T}$-symmetric optical structures," Phys. Rev. A 82, 043803 (2010).

[72] B. Peng, Şahin Kaya Özdemir, F. Lei, F. Monifi, M. Gianfreda, G. L. Long, S. Fan, F. Nori, C. M. Bender, and L. Yang, "Parity-time-symmetric whispering-gallery microcavities," Nature Physics 10, 394-398 (2014).

[73] L. Fan, J. Wang, L. T. Varghese, H. Shen, B. Niu, Y. Xuan, A. M. Weiner, and M. Qi, "An All-Silicon Passive Optical Diode," Science 335, 447-450 (2012).

[74] L. Fan, L. T. Varghese, J. Wang, Y. Xuan, A. M. Weiner, and M. Qi, "Silicon optical diode with 40dB nonreciprocal transmission," Opt. Lett., OL 38, 1259-1261 (2013). 
[75] B. Anand, R. Podila, K. Lingam, S. R. Krishnan, S. Siva Sankara Sai, R. Philip, and A. M. Rao, "Optical Diode Action from Axially Asymmetric Nonlinearity in an All-Carbon SolidState Device," Nano Lett. 13, 5771-5776 (2013).

[76] A. M. Mahmoud, A. R. Davoyan, and N. Engheta, "All-passive nonreciprocal metastructure," Nature Communications 6, 8359 (2015).

[77] D. E. Fernandes and M. G. Silveirinha, "Asymmetric Transmission and Isolation in Nonlinear Devices: Why They Are Different," IEEE Antennas and Wireless Propagation Letters 17, 1953-1957 (2018).

[78] Y. Shi, Z. Yu, and S. Fan, "Limitations of nonlinear optical isolators due to dynamic reciprocity," Nature Photonics 9, 388-392 (2015).

[79] A. B. Khanikaev and A. Alù, "Nonlinear dynamic reciprocity," Nature Photonics 9, 359-361 (2015).

[80] O. Sydoruk, E. Shamonina, V. Kalinin, and L. Solymar, "Terahertz instability of surface optical-phonon polaritons that interact with surface plasmon polaritons in the presence of electron drift," Physics of Plasmas 17, 102103 (2010).

[81] T. A. Morgado and M. G. Silveirinha, "Negative Landau Damping in Bilayer Graphene," Phys. Rev. Lett. 119, 133901 (2017).

[82] T. A. Morgado and M. G. Silveirinha, "Reply to Comment on Negative Landau Damping in Bilayer Graphene," Phys. Rev. Lett. 123, 219402 (2019).

[83] C. G. Poulton, R. Pant, A. Byrnes, S. Fan, M. J. Steel, and B. J. Eggleton, "Design for broadband on-chip isolator using stimulated Brillouin scattering in dispersion-engineered chalcogenide waveguides," Opt. Express, OE 20, 21235-21246 (2012).

[84] M. Hafezi and P. Rabl, "Optomechanically induced non-reciprocity in microring resonators," Opt. Express, OE 20, 7672-7684 (2012)

[85] S. M. Sze, Y. Li, and K. K. Ng, Physics of Semiconductor Devices, 4th ed. (Wiley, Hoboken, NJ, USA, 2021).

[86] A. Yariv and P. Yeh, Optical waves in crystals: propagation and control of laser radiation (Wiley, New York, 1984).

[87] E. Rosencher, A. Fiore, B. Vinter, V. Berger, P. Bois, and J. Nagle, "Quantum Engineering of Optical Nonlinearities," Science 271, 168-173 (1996). 
[88] D. A. Kleinman, "Nonlinear Dielectric Polarization in Optical Media," Phys. Rev. 126, 1977-1979 (1962).

[89] R. W. Boyd, Nonlinear Optics, 3rd ed. (Academic Press, Inc., USA, 2008).

[90] C. Altman and K. Suchy, Reciprocity, Spatial Mapping and Time Reversal in Electromagnetics, 2nd ed. (Springer, 2011).

[91] A. Cerjan and S. Fan, "Achieving Arbitrary Control over Pairs of Polarization States Using Complex Birefringent Metamaterials," Phys. Rev. Lett. 118, 253902 (2017)

[92] See the Supplemental Material for the derivation of (i) the transmission matrix of the MOSFET-metamaterial, (ii) the transmission coefficients of the isolator, and the characterization of the energy transfer between the wave and the material with (iii) macroscopic and (iv) microscopic approaches.

[93] X. Mei, W. Yoshida, M. Lange, J. Lee, J. Zhou, P.-H. Liu, K. Leong, A. Zamora, J. Padilla, S. Sarkozy, R. Lai, and W. R. Deal, "First Demonstration of Amplification at $1 \mathrm{THz}$ Using 25-nm InP High Electron Mobility Transistor Process," IEEE Electron Device Letters 36, 327-329 (2015).

[94] S. H. Wemple and M. DiDomenico, "Electrooptical and Nonlinear Optical Properties of Crystals," in Applied Solid State Science, Vol. 3, edited by Raymond Wolfe (Elsevier, 1972) pp. 263-383. 


\title{
Supplemental Material for the Manuscript: "Nonreciprocal and non-Hermitian material response inspired by semiconductor transistors"
}

\author{
Sylvain Lannebère ${ }^{1}$, David E. Fernandes ${ }^{1}$, Tiago A. Morgado ${ }^{1}$, and Mário G. Silveirinha* \\ 1 Instituto de Telecomunicações and Department of Electrical Engineering, \\ University of Coimbra, 3030-290 Coimbra, Portugal and \\ ${ }^{2}$ University of Lisbon - Instituto Superior Técnico and Instituto de Telecomunicações, \\ Department of Electrical Engineering, 1049-001 Lisboa, Portugal
}

\begin{abstract}
The supplemental material reports (I) the transmission matrix of a MOSFET-metamaterial for normal incidence, (II) the transmission coefficients of the isolator, (III) and (IV) the study of the energy exchanged between the material and the wave, based on macroscopic and microscopic considerations, respectively.
\end{abstract}

\section{TRANSMISSION MATRIX FOR A MOSFET-METAMATERIAL SLAB}

The transmission matrix for a metamaterial slab of thickness $d$ standing in air can be obtained in a standard way using mode matching. For the case of normal incidence $(\mathbf{k}=k \hat{\mathbf{y}})$, using Eq. (7) of the main text, one can write the electric fields in each region as

$$
\mathbf{E}= \begin{cases}\left(\mathbf{1}_{2 \times 2} \mathrm{e}^{i k y}+\bar{R} \mathrm{e}^{-i k y}\right) \cdot \mathbf{E}_{\mathrm{t}}^{\mathrm{inc}} & y<0 \\ \mathbf{E}_{o, 0}\left[A_{o,+} \mathrm{e}^{i k_{o} y}+A_{o,-} \mathrm{e}^{-i k_{o} y}\right]+\mathbf{E}_{e, 0}\left[A_{e,+} \mathrm{e}^{i k_{e} y}+A_{e,-} \mathrm{e}^{-i k_{e} y}\right] & 0 \leq y \leq d \\ \bar{T} \cdot \mathbf{E}_{\mathrm{t}}^{\text {inc }} \mathrm{e}^{i k(y-d)} & y>d\end{cases}
$$

where $A_{o, \pm}, A_{e, \pm}$ are the amplitude of the left/right propagating ordinary and extraordinary waves respectively, $\bar{R}$ and $\bar{T}$ relate the transverse amplitudes of the reflected and transmitted fields, and $\mathbf{E}_{o, 0}=\hat{\mathbf{x}}, \mathbf{E}_{e, 0}=\frac{\varepsilon_{x z}}{\varepsilon_{z z}-\varepsilon_{x x}} \hat{\mathbf{x}}+\hat{\mathbf{z}}$ are two vectors proportional to the system eigenvectors defined in Eq. (6) of the main text. Similarly, the magnetic field can be written as

$$
\mathbf{H}=\frac{1}{\omega \mu_{0}} \mathbf{J} \cdot \begin{cases}k\left(\mathbf{1}_{2 \times 2} \mathrm{e}^{i k y}-\bar{R} \mathrm{e}^{-i k y}\right) \cdot \mathbf{E}_{\mathrm{t}}^{\mathrm{inc}} & y<0 \\ k_{o} \mathbf{E}_{o, 0}\left(A_{o,+} \mathrm{e}^{i k_{o} y}-A_{o,-} \mathrm{e}^{-i k_{o} y}\right)+k_{e} \mathbf{E}_{e, 0}\left(A_{e,+} \mathrm{e}^{i k_{e} y}-A_{e,-} \mathrm{e}^{-i k_{e} y}\right) & 0 \leq y \leq d \\ k \bar{T} \cdot \mathbf{E}_{\mathrm{t}}^{\text {inc }} \mathrm{e}^{i k(y-d)} & y>d\end{cases}
$$

where $\mathbf{J}=\left(\begin{array}{cc}0 & -1 \\ 1 & 0\end{array}\right)$. By matching the fields in (S1) and (S2) at $y=0$ and $y=d$ we obtain a system of linear equations whose solutions give after simplification equation (9) of the main text.

\section{TRANSMISSION COEFFICIENTS OF THE ISOLATOR}

Consider the isolator depicted in Fig.3 (a) of the main text. Without loss of generality, the incident field may be assumed polarized along the direction perpendicular to the input polarizing grid (which depends on the direction of arrival of the incoming wave). Assuming ideal polarizing grids, the right to left transmission coefficient of the isolator is $T_{\text {iso }}^{r \rightarrow l}=T_{21}$, which according to Eq.(9) of the main text is exactly zero. On the other hand, the left to right transmission coefficient of the isolator $T_{\text {iso }}^{l \rightarrow r}=T_{12}$ is precisely:

$$
\left|T_{\text {iso }}^{l \rightarrow r}\right|=\left|\frac{\varepsilon_{x z}}{\varepsilon_{x x}-\varepsilon_{z z}}\left(\gamma_{o}-\gamma_{e}\right)\right|
$$

* To whom correspondence should be addressed: mario.silveirinha@co.it.pt 
Note that the transmission coefficient is non-zero only when $\varepsilon_{x z} \neq 0$.

For propagation along the $+y$ direction (left-to-right), it is possible to get an analytic estimate of $\left|T_{\text {iso }}^{l \rightarrow r}\right|$ by neglecting the reflections at the air-metamaterial interfaces. The incident field polarized along $z$ excites a superposition of the ordinary and extraordinary waves, that according to Eq.(7) of the main text satisfy $A_{o}+A_{e}\left(\frac{\varepsilon_{x z}}{\varepsilon_{z z}-\varepsilon_{x x}}\right)=0$. Thereby, the ordinary and extraordinary wave amplitudes are in phase and the electric field in the metamaterial is:

$$
\mathbf{E}(y)=A_{e}\left(2 i \frac{\varepsilon_{x z}}{\varepsilon_{z z}-\varepsilon_{x x}} \mathrm{e}^{i \frac{k_{e}+k_{o}}{2} y} \sin \left(\frac{k_{e}-k_{o}}{2} y\right) \hat{\mathbf{x}}+\hat{\mathbf{z}} \mathrm{e}^{i k_{e} y}\right)
$$

We note in passing that typically the electric field in the metamaterial is elliptically polarized. The transmission coefficient of the isolator may be approximated by $\left|T_{\text {iso }}^{l \rightarrow r}\right| \approx|\mathbf{E}(d) \cdot \hat{\mathbf{x}}| /|\mathbf{E}(0) \cdot \hat{\mathbf{z}}|$ with $\mathbf{E}$ given by (S4). After some simplifications, one obtains equation (10) of the main text.

As seen in Fig.3 (b) of the main text, this approximation is quite good and explains the origin of the small amplitude oscillations observed in the exact transmission curve: they are due to the standing wave in the metamaterial stemming from the reflections at the second interface.

The behavior of $\left|T_{\text {iso }}^{l \rightarrow r}\right|$ is closely linked to the behavior of the Poynting vector in the metamaterial. Under the same approximations (no reflections), the Poynting vector (Eq.(8) of the main text) in the isolator for left-to-right propagation is

$$
\mathbf{S}=\frac{\left|A_{e}\right|^{2}}{2} \frac{\sqrt{\varepsilon_{0}}}{\sqrt{\mu_{0}}}\left(\sqrt{\varepsilon_{z z}}+\left|\frac{\varepsilon_{x z}}{\varepsilon_{z z}-\varepsilon_{x x}}\right|^{2}\left(\sqrt{\varepsilon_{x x}}+\sqrt{\varepsilon_{z z}}\right)\left(1-\cos \left[\left(k_{e}-k_{o}\right) y\right]\right)\right) \hat{\mathbf{y}}
$$

The first minimum value occurs at $y=0$ and, as $y$ increases, the Poynting vector increases monotonically leading to an enhancement of the power transported in the metamaterial (see Fig.2 of the main text). Interestingly the first maximum occurs at the same distance $d=d_{\max }$ where the transmission is maximized (compare Figs.2 and 3-(b)(i) of the main text).

\section{EXCHANGE OF ENERGY BETWEEN THE WAVE AND THE MATERIAL: MACROSCOPIC APPROACH}

Let us consider a nondispersive lossless material described by the linear permittivity tensor

$$
\bar{\varepsilon}=\left(\begin{array}{ccc}
\varepsilon_{x x} & 0 & \varepsilon_{x z} \\
0 & \varepsilon_{y y} & 0 \\
0 & 0 & \varepsilon_{z z}
\end{array}\right)
$$

The instantaneous power transferred from the wave to the metamaterial is [S1]

$$
Q=\int \mathbf{j}(t) \cdot \mathbf{E}(t) d V
$$

with $\mathbf{j}(t)=\partial_{t} \mathbf{P}(t)$ the polarization current. In time-harmonic regime the electric field is given by $\mathbf{E}(t)=$ $\frac{1}{2}\left(\mathbf{E}_{\omega} \mathrm{e}^{-i \omega t}+\mathbf{E}_{\omega}^{*} \mathrm{e}^{i \omega t}\right)$ and the polarization vector is $\mathbf{P}(t)=\varepsilon_{0}\left(\bar{\varepsilon}-\mathbf{1}_{3 \times 3}\right) \cdot \mathbf{E}(t)$. Then, the time-averaged power can be written as:

$$
\langle Q\rangle=\frac{\omega \varepsilon_{0}}{4} \int i \mathbf{E}_{\omega}^{*} \cdot\left(\bar{\varepsilon}^{\dagger}-\bar{\varepsilon}\right) \cdot \mathbf{E}_{\omega} d V
$$

and is nonzero only if the permittivity tensor is non-Hermitian [S2, $[\mathbf{S 3}]$.

For the particular case of a material described by the permittivity tensor (S6) (with real-valued permittivity) one gets

$$
\langle Q\rangle=\frac{\omega \varepsilon_{0} \varepsilon_{x z}}{2} \int \operatorname{Im}\left\{E_{\omega, x}^{*} E_{\omega, z}\right\} d V
$$

As seen the net power exchanged between the wave and the material is proportional to $\varepsilon_{x z}$ and its sign depends on the relative phase between the components $x$ and $z$ of the electric field. 


\section{EXCHANGE OF ENERGY BETWEEN THE WAVE AND THE MATERIAL: MICROSCOPIC APPROACH}

Let us now consider a nonlinear material with a symmetric electric susceptibility $\left(\bar{\chi}=\bar{\chi}^{T}\right)$ that depends linearly on the electric field $\bar{\chi}=\bar{\chi}(\mathbf{E})$. For such a material the polarization vector in the time-domain is $\operatorname{simply} \mathbf{P}(t)=\varepsilon_{0} \bar{\chi} \cdot \mathbf{E}(t)$ [S4]. From (S7) it follows that the instantaneous power transferred from the wave to the material is

$$
Q=\varepsilon_{0} \int \frac{\partial}{\partial t}[\bar{\chi} \cdot \mathbf{E}] \cdot \mathbf{E} d V
$$

Since by hypothesis $\bar{\chi}$ is a symmetric tensor, the transferred power can be written as

$$
Q=\frac{\varepsilon_{0}}{2} \frac{d}{d t}\left(\int[\mathbf{E} \cdot \bar{\chi} \cdot \mathbf{E}] d V\right)+\frac{\varepsilon_{0}}{2} \int \mathbf{E} \cdot \frac{\partial \bar{\chi}}{\partial t} \cdot \mathbf{E} d V
$$

Here the susceptibility depends implicitly on time because of the nonlinearity:

$$
\frac{\partial \bar{\chi}}{\partial t}=\sum_{k=x, y, z} \frac{\partial E_{k}}{\partial t} \frac{\partial \bar{\chi}}{\partial E_{k}}
$$

In stationary (time-harmonic) regime, the time average of the first term in Eq. S11 is zero. Then, we can write:

$$
\langle Q\rangle=\frac{\varepsilon_{0}}{2}\left\langle\int \mathbf{E} \cdot \frac{\partial \bar{\chi}}{\partial t} \cdot \mathbf{E} d V\right\rangle .
$$

Next we decompose the electric field as $\mathbf{E}=\mathbf{E}_{0}+\mathbf{E}_{\mathrm{AC}}$ with $\mathbf{E}_{0}$ a static field and $\mathbf{E}_{\mathrm{AC}}$ a time-harmonic field oscillating at the frequency $\omega$. The time average operation suppresses all the oscillating terms, so that after simplifications one obtains:

$$
\begin{aligned}
\langle Q\rangle & \approx \varepsilon_{0}\left\langle\int \mathbf{E}_{\mathrm{AC}} \cdot \frac{\partial}{\partial t}\left[\bar{\chi} \cdot \mathbf{E}_{0}\right] d V\right\rangle \\
& \approx\left\langle\int \mathbf{E}_{\mathrm{AC}} \cdot \mathbf{j}^{\mathrm{AC}-\mathrm{DC}} d V\right\rangle
\end{aligned}
$$

Comparing this result with equations (S7) and $(\underline{\mathrm{S} 10})$, we see that $\mathbf{j}^{\mathrm{AC}-\mathrm{DC}}=\varepsilon_{0} \partial_{t}\left[\bar{\chi} \cdot \mathbf{E}_{0}\right]$ defines a dynamic polarization current that is at the origin of the power exchanged between the time-harmonic field and the material. This polarization current is induced by the nonlinearity and by the static electric field.

For the MOSFET metamaterial, the susceptibility is given by Eq. (1) of the main text, so that only the $\chi_{x x}$ component depends on the electric field. From Eq. (S12) a $z$-oscillating field induces a polarization current along the $x$-direction:

$$
\mathbf{j}^{\mathrm{AC}-\mathrm{DC}}=\varepsilon_{0} \frac{\partial \chi_{x x}}{\partial E_{z}} \frac{\partial E_{z}}{\partial t} E_{0 x} \hat{\mathbf{x}}
$$

where $E_{0 x}=\mathbf{E}_{0} \cdot \hat{\mathbf{x}}$. Hence, writing $\mathbf{E}_{\mathrm{AC}}=\frac{1}{2}\left(\mathbf{E}_{\omega} \mathrm{e}^{-i \omega t}+\mathbf{E}_{\omega}^{*} \mathrm{e}^{i \omega t}\right)$ the net power exchanged between the wave and the material becomes:

$$
\langle Q\rangle \approx \frac{\varepsilon_{0} \omega}{2} \frac{\partial \chi_{x x}}{\partial E_{z}} E_{0 x} \int \operatorname{Im}\left\{E_{\omega, x}^{*} E_{\omega, z}\right\} d V .
$$

As shown in the main text the linearization of the electromagnetic response leads to $\varepsilon_{x z}=\frac{\partial \chi_{x x}}{\partial E_{z}} E_{0 x}$. The above formula coincides with Eq. (S9), showing that the microscopic and macroscopic approaches yield precisely the same result.

\section{REFERENCES}


[S2] L. Landau and E. Lifshitz, Electrodynamics of Continuous Media.

[S3] V. M. Agranovich and V. Ginzburg, Crystal Optics with Spatial Dispersion, and Excitons, edited by M. Cardona, P. Fulde, and H.-J. Queisser, Springer Series in Solid-State Sciences, Vol. 42 (Springer Berlin Heidelberg, Berlin, Heidelberg, 1984).

[S4] R. W. Boyd, Nonlinear Optics, Third Edition, 3rd ed. (Academic Press, Inc., USA, 2008). 